\title{
Comparative effectiveness of Low Level Laser therapy and Transcutaneous Electric Nerve Stimulation on Temporomandibular Joint Disorders
}

\author{
Massoud Seifi ${ }^{1}$, Asghar Ebadifar ${ }^{2}$, Sattar Kabiri' ${ }^{1}$, Mohammad Reza Badiee ${ }^{3 *}$, Zahra Abdolazimi ${ }^{4}$, Parisa Amdjadi ${ }^{5}$ \\ 'Department of Orthodontics, School of Dentistry, Shahid Beheshti University of Medical Sciences, Tehran, Iran \\ ${ }^{2}$ Dentofacial Deformities Research Center, Research institute of Dental Sciences, Shahid Beheshti University of Medical \\ Science, Tehran, Iran \\ ${ }^{3}$ Dentofacial Deformities Research Center, Research institute of Dental Sciences, Shahid Beheshti University of Medical \\ Science, Tehran, Iran \\ ${ }^{4}$ Pediatric Department, School of Dentistry, Shahid Beheshti University of Medical Sciences, Tehran, Iran \\ ${ }^{5}$ Department of Dental Materials, School of Dentistry, Shahid Beheshti University of Medical Sciences, Tehran, Iran
}

\author{
*Correspondence to \\ Mohammad Reza Badiee, \\ Dentofacial Deformities Research \\ Center, Dental School, Shahid \\ Beheshti University of Medical \\ Sciences, Tehran, Iran. \\ Tel: +98 2122427752; \\ Fax: +982122427753 \\ Email: \\ mohammadreza.badiee@yahoo.com
}

Published online 29 August 2017

\begin{abstract}
Introduction: Temporomandibular joint disorders (TMDs) are the most common source of pain on the face. There are multiple etiologies, and several types of treatment have been reported. The use of non-invasive and reversible therapies in the treatment of such problems is recommended. The present study evaluated the effect of low-level laser (LLL) therapy and transcutaneous electric nerve stimulation (TENS) on TMDs.

Methods: In this single-blind study, 40 patients with temporomandibular disorders were randomly divided into four groups: TENS (TENSTem dental), LLL (diode $810 \mathrm{~nm} \mathrm{CW}$ ), shamTENS, and sham-LLL. All subjects were examined and data on pain and tenderness in the temporomandibular joint (TMJ) and masticatory muscles (using the visual analogue scale) and mouth-opening (distance between incisal edges before feeling pain; $\mathrm{mm}$ ) were collected before baseline (T1), after each session (T2-T5) and one month after the end of the sessions (T6)), and analyzed using repeated measure analysis of variance (ANOVA) and Bonferroni statistical tests. A $P$ value $<0.05$ was considered significant.

Results: The decrease in pain $(P=0.000)$, tenderness $(P=0.000)$ and increase in mouth-opening ability $(P=0.002)$ was greater in the TENS and LLL groups than in the placebo groups. At the one-month follow-up, significant decrease in pain and tenderness was recorded in the TENS and LLL groups $(P=0.000)$. There was no significant differences between TENS and LLL and the placebo groups for maximum mouth-opening at the end of the study $(P=0.692)$.

Conclusion: Using TENS or LLL therapy can improve TMD symptoms at least for the short term. Although the effects of the placebo played a role in improving symptoms, their effects were less important.

Keywords: Temporomandibular disorders; Low-level laser therapy; Transcutaneous electrical nerve stimulation.
\end{abstract}

\section{Introduction}

Temporomandibular joint disorder (TMD) is a general term for clinical problems in temporomandibular joints (TMJs), masticatory muscles, and surrounding tissues, which manifest as limitations in motion and joint sounds. ${ }^{1}$ TMD is the second most-common skeletal- muscular problem and one of the most common chronic problems involving orofacial pain, discomfort and disability. Approximately $75 \%$ of the population has several symptoms of TMD and 33\% have at least one symptom. ${ }^{2-5}$ The etiology of TMD, especially muscle pain, is multifactorial and includes parafunctional habits, trauma, stress, heredity and occlusal factors ${ }^{5}$; thus, conservative and reversible treatment, especially during initial periods, is recommended. ${ }^{5,6}$ Several treatments have been suggested to control pain and symptoms. These include orthopedic stabilization, intraoral devices, medications (analgesics, muscle relaxants, antidepressants, and placebos) and physical therapy. ${ }^{5}$ The aim of physical therapy is muscle relaxation, a decrease in pain, spasms, swelling and inflammation, and joint stability. ${ }^{7}$ Massage, heat therapy, ultrasound, laser, and transcutaneous 
electric nerve stimulation (TENS) have been used to control symptoms of TMD.?

TENS is a relatively effective, economical, safe, and noninvasive method of reducing acute and chronic pain. ${ }^{8}$ The device causes relaxation of hyperactive muscles and decreases or eliminates pain through the application of electrical current via electrodes attached to the skin. Several theories have been advanced for its effectiveness. Direct stimulation of motor nerves can cause rhythmic contractions in muscles that increases blood flow and decreases muscle edema and hypoxia, which subsequently reduces pain. A second theory is based on the gate control theory in which the application of electrical current or pressure to afferent nerve fibers having larger diameters decreases or prevents the sending of impulses from afferent nerve fibers having smaller diameters by closing the pain gates in the spinal cord. ${ }^{8}$

The use of low-level laser (LLL) therapy has been growing because it is analgesic and anti-inflammatory. It usually excites tissues marked in red during treatment of musculoskeletal disorders with different radiation doses at infrared wavelengths. ${ }^{9,10}$ Its pain control mechanism is not well known; reports suggest that this may occur due to increased release of endogenous epions, improvement of area microcirculation, or an increase in lymphatic flow which reduces edema, increases the production of ATP, and reduces the permeability of nerve cells membranes. ${ }^{9,11,12}$

Several studies have been conducted on the application of TENS and LLL therapy in patients with TMDs with conflicting results. The difference may result from various lengths of application, radiation dose, duration, or the parameters used to assess improvement. Kamyszek et al demonstrated that the use of TENS can significantly decrease muscle activity in patients with TMDs. ${ }^{13}$ Treacy et al showed that muscular awareness relaxation training (MART) was more effective in reducing muscle activity and improving mouth-opening. ${ }^{14}$ Núñez et al revealed that mouth-opening in patients with TMDs increased following application of LLL therapy and TENS. ${ }^{15}$ Mazzetto et al showed that LLL therapy can be used as a supportive treatment in patients with TMDs to reduce pain and improve movement. ${ }^{11}$ The present study evaluated the effectiveness of LLL therapy and TENS on TMDs.

\section{Materials and Methods}

This single-blind clinical trial was conducted with patients referred to the School of Dentistry at Shahid Beheshti University of Medical Sciences, complaining of TMDs. After examination in accordance with the method presented by Dworkin et al, ${ }^{16} 40$ patients (aged 18-50 years) presenting TMDs for which the source muscles were determined, were randomly divided into 4 groups. All patients complained of head and neck pain and tenderness on palpation, especially around the ears and during function, and showed limited mouth-opening. Patients with a history of recent trauma, dental pain, bleeding in the area, and neoplasia or systemic disease involving joints, such as osteoarthritis, rheumatoid arthritis, and diabetes, were excluded. Those with cardiac arrhythmia or pacemakers and pregnant patients were also excluded. Patients who had been receiving other treatments were asked to cease treatment one month before the start of the study.

The purpose of the study, the use of the equipment, its benefits and risks and the chances of being in the control or study group were explained to the patients and they signed consent forms. They were then randomly divided into 4 groups (2 control and 2 study groups with 10 patients per group). All subjects were examined and tenderness of the TMJ and masticatory muscles, including the masseter, temporalis, internal and external pterygoid (using the visual analog scale; VAS) and mouth-opening (distance between incisal edges until feeling pain; $\mathrm{mm}$ ) were recorded.

In the TENS group, a TENSTem dental device (Schwamedico BV; The Netherlands) was applied via electrodes to the skin for 30 minutes at $500 \mathrm{~W}$, a maximum frequency of $50 \mathrm{~Hz}$ and $15 \mathrm{~mA}$ output current. Patients sat in a comfortable chair in a quiet room during intervention. The methods used were those from Wessberg et $\mathrm{al}^{17}$ and Geissler and $\mathrm{McPhee}^{18}$ as well as manufacturer instructions. A positive electrode was placed in the TMJ area ahead of and below the ear hole and an inert electrode was applied to the back of the neck. The patients were instructed to select the power of the device based on their own sensitivity and tolerance so that the process was not painful. ${ }^{19}$ In the sham-TENS group, the same procedure was applied, but the device was turned off.

In the LLL group, a gallium-aluminum-arsenide diode source (Doctor Smile Diode Laser; Italy) with a wavelength of $810 \mathrm{~nm}$, a continuous $0.5 \mathrm{~W}$ peak power output beam and a 5-mm spot size was used. The total amount of irradiation time per painful point was 60 seconds. In the sham-LLL group, the same procedure was followed at the same setting but the device was turned off. The intervention period was four half-hour sessions per week. In order to assess pain and the tenderness of masticatory muscles, VAS was used as the baseline (T1), and after each session (T2-T5). Mouth-opening was recorded in the same manner for all groups. One month after completion of the sessions, the patients were examined to determine the sensitivity in the masticatory muscles and TMJ and maximum mouth-opening, and the results were recorded (T6). Data was evaluated using SPSS (version 21) and the repeated measure analysis of variance (ANOVA), as well as Bonferroni tests (multiple comparisons between groups and times were performed using Bonferroni adjustment) were carried out.

\section{Results}

Kolmogorov-Smirnov statistical analysis showed that the variables examined followed a normal distribution. One-way ANOVA demonstrated that maximum mouth-opening $(P=0.884)$, muscle pain $(P=0.484)$ and 
tenderness $(P=0.933)$ were matched before the study, and no significant difference was observed between the groups.

\section{Maximum Mouth-Opening}

In all groups, mouth-opening improved over time so that maximum mouth-opening was recorded after the last intervention (T5); however, after one month, the improvement compared to baseline was not significant $(P=0.192)$. There was no significant difference between the LLL and TENS groups $(P=0.820)$ at all stages (T1T6). The effectiveness of the placebos were also identical and no significant difference was observed between the groups $(P=0.738)$ (Table 1$)$. Mouth-opening in the intervention groups (TENS and LLL) was significantly greater than for the placebo groups after T3 $(P=0.002)$, but not by the one-month follow-up $(P=0.692)$.

Pain

In the intervention groups (TENS and LLL) pain significantly decreased after T2 and continued until the one month follow-up $(P=0.000)$. The greatest decrease in pain was achieved by the TENS group after T5; however, the difference was not significant $(P=0.12)$. In the placebo groups, pain significantly decreased after T4; however, at the one-month follow-up, there was no significant difference with baseline $(P=0.692$; Table 1$)$.

Tenderness of Masticatory Muscles and TMJ Area Patients in the TENS and LLL groups recorded significantly less tenderness after the first intervention (T2), which continued to the one-month follow-up (T6; $P=0.000$ ). The greatest decrease in tenderness was achieved by the TENS group after T5; however, the difference between groups was not significant at all stages $(P=0.08)$. In the sham groups, the least amount of tenderness was recorded after $\mathrm{T} 5$, and there was no significant difference with the baseline (T1) at the 30-day follow-up $(P=0.83)$. After intervention, the decrease in tenderness in the intervention groups was greater than for the sham groups at all stages $(P=0.000)$.

\section{Discussion}

Various methods have been reported for the management and treatment of TMDs; however, the use of noninvasive interventions is recommended in most cases. ${ }^{20}$ The TENS device is often used in dentistry to control chronic pain and relax masticatory muscles. It is also used in the treatment of trigeminal neuralgia, migraine, and muscle contractions. ${ }^{21}$ In recent years, LLL therapy has been used for the management of musculoskeletal pain, wound healing, and inflammation. ${ }^{22}$

The psychological aspects of TMJ problems treatment are important; the effect of placebos in treatment can be effective in over $40 \%$ of patients. ${ }^{23}$ For this reason, the present study used separate groups with almost the same pre-treatment conditions as placebo groups. Several studies have examined the effectiveness of LLL and TENS for patients with TMD. Wessberg et al, ${ }^{17}$ Møystad et al, ${ }^{24}$ Hansson and Ekblom, ${ }^{25}$ Wieselmann-Penkner et al, ${ }^{26}$ Lewis et $\mathrm{al}^{27}$ and Marchand et $\mathrm{al}^{28}$ discussed the effectiveness of TENS in pain reduction and improving joint symptoms and other muscle aches. Several studies showed that LLL can reduce the pain in TMD patients. ${ }^{4,10-12,22,29,32-35}$

Maximum mouth-opening, pain, and muscle tenderness were the criteria recorded in the present study. After the last session, mouth-opening had significantly decreased in all groups. The greatest improvement occurred in the TENS group (29.11\% recovery), followed by the LLL (24.7\%), sham-TENS (11.33\%) and sham-LLL (8.27\%)

Table 1. Mean (Standard Deviation) of Maximum Mouth Opening, Pain and Tenderness in Millimeters Separated in Different Groups of Intervention

\begin{tabular}{|c|c|c|c|c|c|c|}
\hline Evaluation & $\begin{array}{c}\text { T1 (Before } \\
\text { Intervention) }\end{array}$ & $\begin{array}{c}\text { T2 (1st } \\
\text { Intervention) }\end{array}$ & $\begin{array}{c}\text { T3 (2nd } \\
\text { Intervention) }\end{array}$ & $\begin{array}{c}\text { T4 (3rd } \\
\text { Intervention) }\end{array}$ & $\begin{array}{c}\text { T5 (4th } \\
\text { Intervention) }\end{array}$ & $\begin{array}{l}\text { T6 (30 Days } \\
\text { Fallow up) }\end{array}$ \\
\hline \multicolumn{7}{|l|}{$\mathrm{MMO}^{*}$} \\
\hline TENS & $35.20(2.48)$ & $38.70(2.29)$ & 40.25 (3.09) & $43.20(2.80)$ & 45.45 (1.93) & $37.30(2.10)$ \\
\hline Sham TENS & 35.30 (1.78) & $37.55(2.35)$ & $38.20(2.39)$ & $39.25(2.61)$ & $39.30(2.020$ & $37.55(1.84)$ \\
\hline LLL & $36.20(2.87)$ & $39.50(2.65)$ & 41.13 (2.95) & $44.92(2.79)$ & $45.15(2.34)$ & $38.11(2.33)$ \\
\hline Sham LLL & $35.90(1.64)$ & 37.25 (1.99) & $37.80(2.21)$ & $38.15(2.29)$ & $38.87(2.23)$ & $36.99(1.34)$ \\
\hline \multicolumn{7}{|l|}{ Pain } \\
\hline TENS & 44.95 (5.74) & $33.17(4.73)$ & $28.80(4.79)$ & 23.55 (3.77) & $14.70(6.50)$ & $32.05(5.64)$ \\
\hline Sham TENS & $43.73(5.16)$ & $39.75(5.32)$ & $38.99(4.72)$ & $36.40(4.71)$ & 36.55 (5.78) & $40.45(4.31)$ \\
\hline LLL & $44.58(4.34)$ & $34.15(5.25)$ & $30.24(3.54)$ & $29.35(3.22)$ & $17.67(5.27)$ & $34.29(4.34)$ \\
\hline Sham LLL & $44.24(3.32)$ & $40.13(3.12)$ & 39.99 (4.18) & $38.10(3.12)$ & $37.15(4.58)$ & $41.26(5.11)$ \\
\hline \multicolumn{7}{|l|}{ Tenderness } \\
\hline TENS & 38.60 (4.05) & $30.35(6.36)$ & $28.35(5.22)$ & $25.85(4.51)$ & $17.80(4.12)$ & $22.55(4.27)$ \\
\hline Sham TENS & $38.70(3.35)$ & $35.35(4.91)$ & $34.25(4.37)$ & 32.45 (5.04) & $32.20(5.46)$ & $36.75(3.52)$ \\
\hline LLL & $39.83(4.32)$ & $32.25(5.67)$ & $30.46(4.89)$ & $28.88(3.76)$ & $20.57(3.310$ & $24.11(4.13)$ \\
\hline Sham LLL & $38.12(2.62)$ & $35.16(3.33)$ & $34.15(3.17)$ & $33.45(3.44)$ & $31.78(4.26)$ & $36.23(3.22)$ \\
\hline
\end{tabular}

Abbreviations: $\mathrm{MMO}$, maximum mouth opening. 
groups. Muscle pain and tenderness were measured by VAS on palpation. The most effective treatment for decreasing pain was the TENS group (67.29\% reduction) followed by the LLL (60.36\%), sham-TENS (16.41\%) and sham-LLL (16.02\%) groups. The greatest decrease in tenderness was also obtained by the TENS $(53.88 \%$ reduction) group followed by LLL (48.11\%), sham-TENS (16.79\%), and sham-LLL (16.63\%) groups. At the end of active treatment, both TENS and LLL were more effective than the placebo groups. These results are consistent with those of Gray et $\mathrm{al}^{36}$ and Lewis et al. ${ }^{27}$ At T4 and T5, TENS was more effective than LLL on pain reduction and muscle tenderness, but this was not statistically significant. A common mechanism such as an increase in regional blood flow and a decrease in the permeability of the nerve cell membrane and gate control theory of pain have been considered for both LLL and TENS devices to explain the similar tissue reactions. ${ }^{8,9,11}$

One month after the last intervention, all parameters were evaluated again. As expected, the improvement rate was lower than for the most recently recorded status. Maximum mouth-opening at the one-month follow-up was statistically similar to baseline in all groups. Pain and tenderness had also increased; however, in both the LLL and TENS groups, there was a significant improvement over the baseline. In the placebo groups, no difference was observed. The multifactorial nature of TMD may explain these changes.

Assessing the reliability of objective criteria is easier than for subjective criteria. Pain and tenderness was reported using the VAS scale by patients (subjective) and mouth-opening was measured using a ruler by clinicians (objective). At the 1-month follow-up for both the LLL and TENS groups, patients had less pain and tenderness, but a lower mouth-opening ability.

The placebo effect played a greater role in treatment, muscle pain and tenderness. At T5, these variables had improved compared to baseline in all groups. Lewis et al demonstrated that at 3 weeks after applying TENS and placebo-TENS, the TENS recovery rate was higher; however, $43 \%$ of patients receiving the placebo also showed a reduction in pain. ${ }^{27}$ In a double-blind study, Abreu Venancio et al evaluated the effect of LLL and placebo on pain, tenderness of masticatory muscles and maximum mouth-opening. Their results showed no significant difference between the two groups. ${ }^{9}$ Hansson and Ekblom, in contrast, found that after the use of TENS, 16 of 42 patients experienced a decrease in pain of more than $50 \%$, while in the sham group, only 2 patients showed a decrease. They stated that the effectiveness of TENS is also influenced by the placebo effect, ${ }^{25}$ which is consistent with the findings of the present study. The placebo effect may result from the use of high-tech devices such as TENS or LLL as well as its application by an expert physician; however, the short-term effects of therapy and regenerative intervention should not be ignored.

The efficacy of TENS and LLL therapy in reducing symptoms of TMD was similar; in other words, both methods were equally effective. Núñez et $\mathrm{al}^{15}$ and Kato et $\mathrm{al}^{37}$ reported the effectiveness of TENS and LLL for TMD, but found that for some parameters, the effect of LLL was greater than for TENS. In the present study, although there was no significant difference, TENS was found to be more effective than LLL. This could result from differences in the radiation dose, duration, and irradiated area. Each intervention had limitations and advantages. Although the use of TENS is easier than LLL because of the smaller size involved, decreased risk and portability of the devise, electrical currents delivered to the skin through the TENS may cause discomfort in some patients.

\section{Conclusion}

The use of TENS or LLL can improve TMD symptoms, at least for the short term. Either TENS or LLL can be considered as an alternative physical modality or supplementary approach for management of temporomandibular disorder. The effects of the placebo in improving symptoms played a limited role. The present study did not evaluated the long term effect of treatment and there is need to design a study with longer follow-ups.

\section{Ethical Considerations}

Ethical considerations were approved by the Institutional Review Board (Ethics Committee).

\section{Conflict of Interests}

None.

\section{References}

1. Randhawa K, Bohay R, Côté P, et al. The effectiveness of noninvasive interventions for temporomandibular disorders: a systematic review by the Ontario Protocol for Traffic Injury Management (OPTIMa) Collaboration. Clin J Pain. 2016;32(3):260-78. doi: 10.1097/AJP.0000000000000247.

2. Dworkin SF, Huggins KH, LeResche L, et al. Epidemiology of signs and symptoms in temporomandibular disorders: clinical signs in cases and controls. J Am Dent Assoc. 1990;120(3):273-281.

3. Emshoff R, Bösch R, Pümpel E, Schöning H, Strobl H. Lowlevel laser therapy for treatment of temporomandibular joint pain: a double-blind and placebo-controlled trial. Oral Surg Oral Med Oral Pathol Oral Radiol Endodontol. 2008;105(4):452-456.

4. Kulekcioglu S, Sivrioglu K, Ozcan O, Parlak M. Effectiveness of low-level laser therapy in temporomandibular disorder. Scand J Rheumatol. 2003;32(2):114-118.

5. Liu F, Steinkeler A. Epidemiology, diagnosis, and treatment of temporomandibular disorders. Dent Clinf North Am. 2013;57(3):465-479. doi:10.1016/j.cden.2013.04.006.

6. Koray O, Küçük B, Edebeoglu B. Etiology of temporomandibular disorder pain. J Orofac Pain. 2009;21(3):89-94.

7. McNeely ML, Olivo SA, Magee DJ. A systematic review of the effectiveness of physical therapy interventions for temporomandibular disorders. Phys Ther. 2006;86(5):710725 .

8. Grossmann E, Tambara JS, Grossmann TK, Siqueira JT. Transcutaneous electrical nerve stimulation for 
temporomandibular joint dysfunction. Revista Dor. 2012;13(3):271-276.

9. Abreu Venancio R, Camparis CM, Fátima Zanirato Lizarelli R. Low intensity laser therapy in the treatment of temporomandibular disorders: a double-blind study. J Oral Rehabil. 2005;32(11):800-807.

10. Carvalho CM, de Lacerda JA, dos Santos Neto FP, Cangussu MCT, Marques AM, Pinheiro AL. Wavelength effect in temporomandibular joint pain: a clinical experience. Lasers Med Sci. 2010;25(2):229-232. doi:10.1007/s10103009-0695-y.

11. Mazzetto MO, Hotta TH, Pizzo RCdA. Measurements of jaw movements and TMJ pain intensity in patients treated with GaAlAs laser. Braz Dent J. 2010;21(4):356-360.

12. De Medeiros JS, Vieira GF, Nishimura PY. Laser application effects on the bite strength of the masseter muscle, as an orofacial pain treatment. Photomed Laser Ther. 2005;23(4):373-376.

13. Kamyszek G, Ketcham R, Garcia R Jr, Radke J. Electromyographic evidence of reduced muscle activity when ULF-TENS is applied to the Vth and VIIth cranial nerves. Cranio. 2001;19(3):162-168.

14. Treacy K. Awareness/relaxation training and transcutaneous electrical neural stimulation in the treatment of bruxism. $J$ Oral Rehabil. 1999;26(4):280-287.

15. Núñez SC, Garcez AS, Suzuki SS, Ribeiro MS. Management of mouth opening in patients with temporomandibular disorders through low-level laser therapy and transcutaneous electrical neural stimulation. Photomed Laser Surg. 2006;24(1):45-49.

16. Dworkin SF, LeResche L, DeRouen T, Von Korff M. Assessing clinical signs of temporomandibular disorders: reliability of clinical examiners. J Prosthet Dent. 1990;63(5):574-579.

17. Wessberg GA, Carroll WL, Dinham R, Wolford LM. Transcutaneous electrical stimulation as an adjunct in the management of myofascial pain-dysfunction syndrome. $J$ Prosthet Dent. 1981;45(3):307-314.

18. Geissler P, McPhee P. Electrostimulation in the treatment of pain in the mandibular dysfunction syndrome. J Dent. 1986;14(2):62-64.

19. Schiffman E, Look J, Hodges J, Swift J, Decker K, Hathaway $\mathrm{K}$, et al. Randomized effectiveness study of four therapeutic strategies for TMJ closed lock. J Dent Res. 2007;86(1):5863.

20. Wright EF, North SL. Management and treatment of temporomandibular disorders: a clinical perspective. J Man Manip Ther. 2009;17(4):247-254.

21. Cooper B. The role of bioelectronic instruments in the management of TMD. N Y State Dent J. 1995;61(9):48-53.

22. Çetiner S, Kahraman SA, Yücetas S. Evaluation of lowlevel laser therapy in the treatment of temporomandibular disorders. Photomed Laser Ther. 2006;24(5):637-641.

23. Dimitroulis G. Temporomandibular disorders: a clinical update. BMJ. 1998;317(7152):190-194.
24. Møystad A, Krogstad BS, Larheim TA. Transcutaneous nerve stimulation in a group of patients with rheumatic disease involving the temporomandibular joint. J Prosthet Dent. 1990;64(5):596-600.

25. Hansson P, Ekblom A. Transcutaneous electrical nerve stimulation (TENS) as compared to placebo TENS for the relief of acute oro-facial pain. Pain. 1983;15(1):157-165.

26. Wieselmann-Penkner K, Janda M, Lorenzoni M, Polansky R. A comparison of the muscular relaxation effect of TENS and EMG-biofeedback in patients with bruxism. J Oral Rehabil. 2001;28(9):849-853.

27. Lewis D, Lewis B, Sturrock RD. Transcutaneous electrical nerve stimulation in osteoarthrosis: a therapeutic alternative? Ann Rheum Dis. 1984;43(1):47-49.

28. Marchand S, Charest J, Li J, Chenard J-R, Lavignolle B, Laurencelle L. Is TENS purely a placebo effect? A controlled study on chronic low back pain. Pain. 1993;54(1):99-106.

29. Fikácková H, Dostálová T, Navrátil L, Klaschka J. Effectiveness of low-level laser therapy in temporomandibular joint disorders: a placebo-controlled study. Photomed Laser Surg. 2007;25(4):297-303. doi: 10.1089/pho.2007.2053

30. Shirani AM, Gutknecht N, Taghizadeh M, Mir M. Low-level laser therapy and myofacial pain dysfunction syndrome: a randomized controlled clinical trial. Lasers Med Sci. 2009;24(5):715-720. doi:10.1007/s10103-008-0624-5.

31. Hotta PT, Hotta TH, Bataglion C, et al. Emg analysis after laser acupuncture in patients with temporomandibular dysfunction (TMD). Implications for practice. Complement Ther Clin Pract. 2010;16(3):158-160. doi:10.1016/j. ctcp.2010.01.002.

32. Khalighi HR, Mortazavi H, Mojahedi SM, Marhabi SA, Abbasabadi FM. Low level laser therapy versus pharmacotherapy in improving myofascial pain disorder syndrome. J Lasers Med Sci. 2016;7(1):45-50. doi:10.15171/ jlms.2016.10.

33. Ahrari F, Madani AS, Ghafouri ZS, Tunér J. The efficacy of low-level laser therapy for the treatment of myogenous temporomandibular joint disorder. Lasers Med Sci. 2014;29(2):551-557. doi:10.1007/s10103-012-1253-6.

34. Salmos-Brito JA, de Menezes RF, Teixeira CE, et al. Evaluation of low-level laser therapy in patients with acute and chronic temporomandibular disorders. Lasers Med Sci. 2013;28(1):57-64. doi:10.1007/s10103-012-1065-8.

35. Marini I, Gatto MR, Bonetti GA. Effects of superpulsed low-level laser therapy on temporomandibular joint pain. Clin J Pain. 2010;26(7):611-616.

36. Gray R, Quayle A, Hall C, Schofield M. Physiotherapy in the treatment of temporomandibular joint disorders: a comparative study of four treatment methods. Br Dent $\mathrm{J}$. 1994;176(7):257-261.

37. Kato MT, Kogawa EM, Santos CN, Conti PCR. TENS and low-level laser therapy in the management of temporomandibular disorders. J Appl Oral Sci. 2006;14(2):130-135. 